Posprint of: Journal of Biotechnology Volume 151, Issue 2, 20 January 2011, Pages 180-185

\title{
Assessment of carotenoid production by Dunaliella salina in different culture systems and operation regimes
}

Ana Prieto (a), J. Pedro Cañavate (a), Mercedes García-González (b)

a IFAPA Centro "El Toruño", Apartado. 16, 11500 Cádiz, Spain

b Instituto de Bioquímica Vegetal y Fotosíntesis, Consejo Superior de Investigaciones Científicas-Universidad de Sevilla, Avda. Américo Vespucio, 49, 41092 Sevilla, Spain

\begin{abstract}
The effect of operation regime and culture system on carotenoid productivity by the halotolerant alga Dunaliella salina has been analyzed. Operation strategies tested included batch and semi continuous regime, as well as a two-stage approach run simultaneously in both, open tanks and closed reactor. The best results were obtained with the closed tubular photobioreactor. The highest carotenoid production (328.8 mg carotenoid I-1 culture per month) was achieved with this culture system operated following the two-stage strategy. Also, closed tubular photobioreactor provided the highest carotenoid contents ( $10 \%$ of dry weight) in Dunaliella biomass and $\beta$-carotene abundance (90\% of total carotenoids) as well as the highest 9-cis to all-trans $\beta$-carotene isomer ratio (1.5 at sunrise).
\end{abstract}

\section{Keywords}

Microalgae; Dunaliella salina; $\beta$-Carotene; Photobioreactor; Biotechnology; Outdoor culture

\section{Introduction}

$\beta$-Carotene is a terpenoid pigment of increasing demand and a wide variety of market applications: as food colouring agent, as pro-vitamin A in food and animal feed, as an additive to cosmetics and multivitamin preparations and as a health food product under the antioxidant claim (Edge et al., 1997 and Johnson and Schroeder, 1995). The most important procedure for the natural production of $\beta$-carotene is the culture of the unicellular biflagellate marine green microalga Dunaliella salina (Borowitzka, 1995). The extent of carotenoids accumulation in oil globules within the interthylakoid spaces of their chloroplast is directly proportional to the integral amount of light to which D. salina cells are exposed during a division cycle (Ben-Amotz and Avron, 1983). Accumulation is enhanced under several conditions: high irradiance, stress temperatures, high salt concentration and/or nutrient deficiency (Ben-Amotz and Shaish, 1992). Under these conditions, up to $10 \%$ of the alga dry weight is $\beta$-carotene (Ben-Amotz, 1999). Dunaliella $\beta$-carotene occurs as a number of isomers, two of which, 9-cis and all-trans (in approximately equal amount), make up approximately $80 \%$ of the total (Borowitzka and Borowitzka, 1988). Superior bioavailability, antioxidant capacity 
and physiological effects, substantiate the commercial interest of the algal carotene over its synthetic counterpart ( Becker, 1994 and Ben-Amotz, 2009).

All existing commercial Dunaliella facilities grow the alga outdoors in open-air cultures at high salinity: extensive culture systems (Australia and China) or more intensive, paddle-wheel stirred raceway ponds (Israel and USA) (Del Campo et al., 2007). Several trials have been initiated to grow Dunaliella in closed photobioreactors, although up to date, none of these trials have taken production beyond the laboratory or small pilot scale plant (García-González et al., 2005).

The production in open systems has many disadvantages which relate to the factors governing outdoor productivity of photoautotrophic microorganisms (Richmond, 1991): the relatively long light-path (pond-depth) which mandates maintenance of relatively dilute cultures difficult to harvest, high CO2 consumption with low efficiency, contamination problems, impractical control of some environmental factors as temperature and the relatively large running costs due to the need of large volumes of water, salt and land. The use of closed tubular photobioreactors represents a very interesting alternative to outdoor open tanks, since they offer high values of both photosynthetic efficiency and productivity (reduced light-path) diminishing the operational inputs ( $\mathrm{CO} 2$, water, salt, nutrients) and providing steady and controlled conditions (Borowitzka, 1999 and Tredici and Zitelli, 1997). However, they are certainly more expensive to build and operate than the open systems.

The carotenoid content of the microalgal biomass is positively correlated with the market price for the latter. Therefore, the influence of the operating conditions on Dunaliella cultures on the quality of its biomass is of major concern. The so-called two stage process entails the dilution of dense batch cultures and the simultaneous limitation in key nutrients, and is a commercial procedure to achieve Dunaliella biomass with high carotenoid content (BenAmotz, 1995). This approach results in low cell density suspensions, with high cost in harvesting cells due to the handling of huge volumes of liquid. Closed tubular photobioreactors allow the management of dense cell populations, which can also have a high carotenoid content if the system is properly operated (García-González et al., 2005). A complementary approach to achieve carotenoid-rich Dunaliella cells might result from the operation of the culture under semi continuous regime (Del Campo et al., 2007).

In this work, the potential of different culture systems (open tanks and tubular reactor) and operation procedures (batch, semi continuous or two-stage cultures) has been assessed simultaneously, with regard to both the carotenoid accumulation by Dunaliella and the isomeric composition of $\beta$-carotene in this biomass, in order to determine the best strategy for high quantity and quality pigment production. The work was performed under the environmental conditions of the Bay of Cádiz (SW Spain), and was extended for several years round cycle.

\section{Materials and methods}

The strain used in this work was D. salina UTEX 2538, from the Culture Collection of Texas University (USA). Inocula for outdoor cultures were prepared on $\mathrm{f} 2$ medium described by Guillard and Ryther (1962). For outdoor cultures, seawater of $12.5 \%$ salinity was sterilized with 
$10 \mathrm{ppm}$ chlorine, neutralized and supplemented with $1.5 \mathrm{mM} \mathrm{NaNO}, 100 \mu \mathrm{M} \mathrm{NaH2PO} 4$ and $12 \mu \mathrm{M} \mathrm{FeCl} 3 \cdot 6 \mathrm{H} 2 \mathrm{O}$.

Outdoor open cultures were performed in the experimental plant of Centro de Investigación de Cultivos de Especies Marinas, located at Puerto Santa María, Cádiz (latitude $36^{\circ} 38^{\prime} \mathrm{N}$, longitude $6^{\circ} 11^{\prime} \mathrm{W}$ ) using four oval fibreglass tanks (ponds) with an open-air surface area of 3 $\mathrm{m} 2$ and a central wall partition to improve culture circulation. Agitation was promoted by an arm paddle-wheel of three paddles operating at a rotating speed of about $19 \mathrm{rpm}$ (cell suspension flow rate, $0.55 \mathrm{~m} \mathrm{~s}-1$ ). Temperature and irradiance were not controlled. The $\mathrm{pH}$ of the culture was permanently adjusted at 8.0 by means of automatic $\mathrm{CO} 2$ injection regulated by a $\mathrm{pH}$-controller. When needed, $\mathrm{CO} 2$ was supplied through porous plastic pipes fixed at the bottom of the tank. A precise gas flow rate of 0.4 I min-1 was achieved using digital mass flow meters. Outdoor closed cultures were carried out in a tubular system similar to previously described by Del Campo et al. (2001), consisting essentially in a 60-I polymethyl metacrylate tubular photobioreactor with an air-lift system to recirculate the cell culture and an horizontal loop, consisting of tubes (90 m long, $2.4 \mathrm{~cm}$ inner diameter and $2.2 \mathrm{~m} 2$ surface), which acts as solar receiver, submerged in a water pond. The air-lift was made up of a degasser (in which the $\mathrm{pH}$ and temperature probes were inserted) and two tubes $3 \mathrm{~m}$ high (the riser and the downcomer). Compressed air was supplied into the riser tube to move the cell suspension through the tubes and provide turbulence. The culture $\mathrm{pH}$ was controlled by the addition of $\mathrm{CO} 2$ gas.

The photosynthetic available irradiance (PAR 400-700 nm) impinging the culture surface was measured using a quantum sensor (model LI-190 SZ), connected to a data logger (model LI1400, Li-Cor Inc., Lincoln, NE, USA).

Inocula for outdoor cultures were prepared in 50 I bubble columns indoor. Cells from several columns were mixed and distributed between tanks and photobioreactor. In cultures operated under batch regime, the cells were grown on complete medium at an initial cell concentration of $0.4 \times 106$ cell ml-1 in open tank or $1.5 \times 106$ cell ml-1 in closed tubular reactor.

For operation under semi-continuous regime, the culture was diluted at intervals through removal of the required volume of the cell suspension and replacement with fresh medium as to reach an established minimal population density (about $0.9 \times 106$ cell ml-1 $(0.13 \mathrm{~g} \mathrm{l}-1)$ in open tank and $3 \times 106$ cell $\mathrm{ml}-1(0.45 \mathrm{~g} \mathrm{l}-1)$ in closed tubular reactor) at the beginning of each cycle; the culture was allowed then grow until reaching $1.5 \times 106$ cell ml-1 in open tank or $9 \times$ 106 cell $\mathrm{ml}-1$ in closed tubular reactor, and the cycle repeated.

In the two-stage batch process, first, the cells were grown on complete medium containing nitrate under optimal conditions to attain a high cell concentration (to $0.9 \times 106$ cell ml-1 in open tank or $5 \times 106$ cell $\mathrm{ml}-1$ in closed tubular reactor), diluting the algal culture thereafter with nitrate-free medium to $0.4 \times 106$ cell ml-1 in open tank or $2.5 \times 106$ cell ml-1 in closed tubular reactor, conditions that induce accumulation of the pigment taking advantage of a synergistic effect of light availability, nitrate starvation and low cell density (Ben-Amotz, 1995). 
Cell density was determined by direct counting, using a light microscope (magnification 400x) with a $0.1 \mathrm{~mm}$ deep counting chamber (Neubauer). Dry weight was determined on pre-washed glass fibre filter with aliquots of the culture, washed with ammonium formiate $1 \%(\mathrm{w} / \mathrm{v})$ and dried at $80^{\circ} \mathrm{C}$ for $24 \mathrm{~h}$. These determinations gave an average value of $153 \pm 3 \mathrm{pg}$ cell-1.

For carotenoid determination, 1-ml aliquot of algal suspension was centrifuged at $1000 \times \mathrm{g}$ for $5 \mathrm{~min}$ and the pellet extracted with $3 \mathrm{ml}$ of ethanol:hexane 2:1 (v/v). Two millilitres of water and $4 \mathrm{ml}$ hexane were added and the mixture vigorously shaken and centrifuged again at 1000 $\times \mathrm{g}$ for $5 \mathrm{~min}$. The hexane layer was separated and its absorbance determined: A450 × 25.2 equal the micrograms of carotene in sample (Shaish et al., 1992).

For $\beta$-carotene isomer analysis, assays were performed using total carotenoid extracts from cells subjected to 7 days of $\mathrm{N}$ starvation, at the end of the dark period ( 8 a.m.) and after $10 \mathrm{~h}$ of sunlight (6 p.m.), in periods of high irradiance. Aliquots of these extracts were evaporated under $\mathrm{N} 2$ at $30{ }^{\circ} \mathrm{C}$, redissolved in methylene chloride and analyzed by HPLC according to the method described by Ben-Amotz et al. (1988), in a Vydac TP201 54 column eluted with isocratic solvent of $1 \mathrm{ml}$ min-1 methanol:acetonitrile $(9: 1, \mathrm{v} / \mathrm{v})$. A High Performance Liquid Chromatograph, Waters LC-module 1 plus, equipped with a spectrophotometric detector, (Waters, Milford, MA, USA) was used.

Biomass or carotenoid accumulation indicated the concentration (in $\mathrm{mg} \mathrm{l}-1$ ) reached at the end of an experiment. The aggregated monthly carotenoid production was calculated as average from the accumulation values obtained in every culture carried out in each period of time (carotenoid production) multiplied by the number of cycles comprised in the full month.

The occurrence of protozoa was microscopically checked daily. Contaminated cultures were treated with quinine sulphate $(10 \mathrm{mg} \mathrm{l}-1)$ for protozoa elimination.

\section{Results}

\subsection{Carotenoid production in open-tanks}

Kinetic for biomass growth in batch regime in spring is shown in Fig. 1A. This season was the most productive reaching values of about $450 \mathrm{mg}$ biomass $\mathrm{I}-1$ and $8.9 \mathrm{mg}$ carotenoids $\mathrm{I}-1$ (Table 1). The annual average was $7.75 \pm 1.02 \mathrm{mg}$ carotenoid $\mathrm{I}-1$ that represented about $3 \%$ of the dry biomass. In winter time, carotenoid production was only $6.93 \pm 1.41 \mathrm{mg}$ carotenoid -1 , this figure representing around $5 \%$ of dry matter. Under this operating regime, maximal biomass and carotenoid accumulation of the culture were recorded after 10-12 days and then it was possible to carry out two culture runs per month. Thus, when considered on monthly basis, aggregated carotenoid production calculated was $17.8 \mathrm{mg} \mathrm{l}-1$ for the best climatic conditions (spring) and $15.5 \mathrm{mg} \mathrm{I}-1$ considering the whole year. The influence of the season on the monthly average carotenoid production values, estimated in four consecutive years of experimentation, is shown in Fig. 2.

During two years, parallel cultures in open tanks were operated under semi-continuous regime, performing 6 dilution cycles per month as described in Section 2. A representative example of the evolution of biomass in a semi-continuous culture in spring is shown in Fig. 1B. In this season, the carotenoid accumulation in the culture was $3.8 \mathrm{mg} \mathrm{I-1} \mathrm{per} \mathrm{production} \mathrm{cycle,}$ 
thus resulting in an aggregated monthly production of about $22.8 \mathrm{mg}$ carotenoid I-1 (Table 1 ), and an average of $14.9 \pm 6.8 \mathrm{mg} \mathrm{I}-1$ when considering two whole years of experimentation. The differences among seasons are shown in Fig. 2. The general response at low temperatures of the semi-continuous cultures with regard to production and carotenoid content of the dry biomass (3\%) were similar to those of batch cultures.

\subsection{Carotenoid production in closed tubular reactor}

About $1.5 \times 106$ cells $\mathrm{ml}-1$ were needed to start successfully the outdoor culture in this system. Equally essential was the coverage of the tubular loop reactor with an appropriate sunshade screen, to protect cells against light-induced damage during the first 3 days (photoadaptation period). Attempts to operate the photobioreactor under continuous regime were unsuccessful, since even the lowest dilution rate allowed by our equipment resulted in the washout of the culture. Kinetic for biomass growth in batch regime for this reactor is shown in Fig. 3A. Maintenance of photobioreactor operation under this regime resulted in maximal biomass accumulation of $2 \mathrm{~g} \mathrm{l}-1$ with a carotenoid accumulation of $90 \mathrm{mg} \mathrm{l}-1$ and minimum with $0.5 \mathrm{~g}$ biomass $\mathrm{I}-1$ and $30 \mathrm{mg}$ carotenoid $\mathrm{I}-1$, both after 8 days of growth ( 3 cycles per month). Fig. 2 shows the aggregated monthly production of carotenoids, recorded throughout two whole years of operation. The average carotenoid accumulation per cycle in spring was $62.2 \mathrm{mg} \mathrm{l}-1$ (Table 1), equivalent to an average value for the aggregated monthly production of $186.5 \mathrm{mg}$ carotenoids I-1 for the two-year operation period. The Dunaliella biomass obtained had a carotenoid content of about $5 \%$ on a dry weight basis.

To operate the tubular reactor under semi-continuous regime, dilutions of the culture at 5 day intervals were performed (Fig. 3B). Under these conditions, an average biomass accumulation of $5.5 \mathrm{~g} \mathrm{l}-1$ with carotenoid accumulation of $50 \mathrm{mg} \mathrm{l}-1$ per cycle was achieved in spring (aggregated monthly production, $250 \mathrm{mg} \mathrm{l}-1$ ) (Table 1). The average carotenoid content of the biomass under these conditions was about $5-6 \%$.

\subsection{Two-stage strategy}

We have analyzed the accumulation of carotenoids during the second phase (with $\mathrm{N}$ availability restriction) in two-stage cultures, both in open tanks and in tubular reactor. All these experiments were carried out in spring.

In open tanks, exponentially growing cultures (about 5 days old) were diluted with $\mathrm{N}$-free medium. Within 6 days, biomass of the culture increased slightly, whereas the carotenoid content, both in the culture and in the biomass, increased substantially achieving $14.3 \pm 0.53$ mg I-1 culture (about $9.7 \%$ of dry biomass). Altogether, three complete two-phase cultures were carried to complete each month involving an aggregated monthly production of $42.9 \mathrm{mg}$ carotenoids I-1 (Table 1). In this system, the extent of carotenoid accumulation in the second stage was directly related to the impinging irradiance (Table 2).

For the tubular reactor, within 5 days after dilution of the culture (four complete two-phase cultures per month) the carotenoid accumulation increased up to $82.2 \pm 2.3 \mathrm{mg} \mathrm{l}-1$ culture (about $10 \%$ of dry biomass) resulting in an aggregated monthly production of $328.8 \mathrm{mg}$ carotenoids $\mathrm{I}-1$ (Table 1 ). 
Representative values obtained for the different strategies along the same period are compiled in Table 1, in order to compare the carotenoid production for each operation protocol, namely batch, semi continuous or two-stage regime in either open tanks or closed photobioreactor. Data considered for the comparison correspond to experiments carried out in spring. Operating the cultures under semi continuous regime provided values for carotenoid productivity about $50 \%$ higher than those obtained with the simple batch option; the application of the two-stage approach resulted in the highest productivity. Closed photobioreactor exhibited the best performance at all instances, with carotenoid productivity values 8 - to 10 -fold higher than those in open tanks. The highest carotenoid production was achieved when the tubular photobioreactor was operated under the two-stage strategy.

\subsection{Isomeric $\beta$-carotene composition}

The levels of total $\beta$-carotene, its 9 -cis and all-trans isomers and lutein in biomass of $D$. salina were analyzed in the second phase of two stage cultures since presented the highest carotenoid percentage, in both open tanks and closed tubular reactor. In open tanks cultures, $\beta$-carotene accounted for about $80 \%$ of total carotenoids, and lutein about $4 \%$. No change in the $\beta$-carotene content was recorded along the daylight period, and a constant 9-cis to alltrans $\beta$-carotene ratio $(1.01 \pm 0.05)$ was maintained in the same time scale (Table 3 ).

In cultures performed in closed tubular reactor, the major carotenoids were also $\beta$-carotene $(90 \%)$ and lutein (5\%). With regard to isomer composition, at sunrise $\beta$-carotene was enriched in the 9-cis isomer, which decreased progressively during the daylight period, with a concomitant increase in the level of the all-trans isomer. This led to a change in the 9-cis to alltrans ratio, from $1.53 \pm 0.08$ (8 a.m.) to $1.04 \pm 0.05$ ( 4 p.m.). After the sunset, the level of alltrans $\beta$-carotene started to decrease, while the 9 -cis isomer increased until the isomer balance found at sunrise (about $60 \%$ 9-cis versus about $40 \%$ all-trans- $\beta$-carotene) was eventually recovered.

\section{Discussion}

Closed photobioreactors are considered to have several major advantages over open tanks for microalgal biomass production (Del Campo et al., 2007 and Wijffels et al., 2009). Nevertheless, still today most mass production commercial facilities operate with open systems. Factors as occupied surface, capital investment, energy costs, labour, water, nutrients, specific alga requirements, local climatic conditions and productivity must be considered. The production cost of algal biomass in closed reactors may be as high as an order of magnitude higher than in tanks (Tredici, 2004). A compromise between all of these factors should guide the final choice of a particular system to achieve an economically acceptable outcome.

Up to date, open tanks represent the conventional method used in commercial production plants for Dunaliella (Borowitzka and Borowitzka, 1988 and Ben-Amotz, 1999). Different production approaches have been recently considered (Del Campo et al., 2007), but a proper efficiency comparison still awaited for data from their simultaneous operation. In this work, the carotenoid production of outdoor batch and semi continuous cultures of Dunaliella in open 
tanks and closed tubular reactor has been compared (Table 1). In open systems operated under batch regime, the average annual production was 10-fold lower and the carotenoid content of the biomass half than those in closed tubular reactors. A smaller light pathway in closed tubular reactors ( Lee, 2001 and Richmond et al., 1993) could explain that better performance. An increase in the $\beta$-carotene content of the cells does not necessarily result in an increase in the volumetric production of $\beta$-carotene; other parameters, such as biomass concentration, are also important (Hejazi et al., 2003). In our tubular reactor, both volumetric biomass production and cellular carotenoid content were higher than those in open tanks. Similar results were found under semi continuous regime, with carotenoid production being 11-fold lower in open tanks than in the closed tubular reactor. Taken together these results point out that tubular reactor should be chosen as culture system.

The cultivation employing a two-stage technology is more productive than batch and semi continuous cultures, both in the tubular reactor and in the open tank, confirming that the twostage cultures is the best approach for carotenoid production by Dunaliella (Ben-Amotz, 1995).

In order to decide the best performance for commercial carotenoid production, other factors that affect the efficiency of each system must also be considered, such as time and energy required for operations, harvesting, cleaning and refilling. The size of the volume to be handled in these operations strongly influences the energy consumption and the final cost. Batch cultures require permanent availability of initial inocula, and their product is not homogeneous. On the other hand, semi continuous cultures are easier to operate and control, although they demand a permanent supply of high volume of fresh medium.

Another critical feature to consider is contamination. Although the high salinity of D. salina cultures restricts development of predators (Borowitzka, 1992), in our outdoor experience, ciliates appeared occasionally in the cultures. In the case of batch cultures in open tanks, the protozoa showed up after about 8 days of operation, their appearance being delayed to about 20 days when the cultures were operated under semi continuous regime, but they were never found in the closed reactor.

The combined effects of light and growth limitation on $\beta$-carotene accumulation led to the proposal that accumulation of the pigment is directly proportional to the integral amount of light to which the D. salina cells are exposed during a division cycle (Ben-Amotz and Avron, 1983). Our results are in agreement with this hypothesis, showing a correlation between light intensity and carotenoid accumulation.

Our results show that the cell content in $\beta$-carotene and lutein is higher in the closed tubular photobioreactor (where light availability is optimal) than in open tanks. Other important difference is the prevalence of the 9-cis isomer in closed reactor cultures that probably also correlates with a higher light availability in this design. The presence of this isomer is considered a quality criterion, given its outstanding antioxidant properties in vivo ( Liu et al., 2000 and Palozza and Krinsky, 1992). Moreover, the simultaneous occurrence of higher levels of lutein and 9-cis $\beta$-carotene in closed tubular reactor increases the value of the biomass obtained in this culture system. 
The influence of the solar cycle on the isomeric composition of $\beta$-carotene has been established. In open tanks, both the $\beta$-carotene concentration and the 9 -cis to all-trans isomer ratio remain virtually constant, exhibiting similar values early and later in the day. In the closed tubular reactor, the $\beta$-carotene concentration was constant along the day, but the 9 -cis to alltrans isomer ratio was higher at sunrise, decreased along the day and recovered during the night period. These results differ from to those reported by Phillips et al. (1995) although irradiance conditions were different, and are in agreement with those of García-González et al. (2005), Gómez and González (2005), Jimenez and Pick (1994) and Orset and Young (2000), who observed a higher 9-cis/all-trans ratio at low irradiance. The discrepancies between references may due to isomerization which can occur during extraction and/or analysis of the pigments (Orset and Young, 2000), as well as to the different culture conditions used in each work.

It has been suggested that light plays a major role in the biosynthesis of 9 -cis- $\beta$-carotene, assuming a photoprotective role of this isomer, and its conversion into the all-trans form in darkness (Ben-Amotz et al., 1988). In our experimental conditions, a preferred synthesis of alltrans isomer or interconversion from 9-cis is observed. The abundance of 9-cis isomer early in the morning enhances the quality of the pigment that can be obtained from cells harvested at this stage.

\section{Conclusions}

The results presented allow to assure that culture of Dunaliella in closed tubular reactor under a two stage strategy shows better performance than open tanks cultures when simultaneously operated outdoor: carotenoid productivity and $\beta$-carotene and lutein contents are much higher in the closed tubular reactor, the $\beta$-carotene obtained is enriched in 9 -cis isomer resulting in a more quality product, and no contamination was detected in this reactor design. Nevertheless, other factors not included in this study as operation and energy cost must also be evaluated since they could restrict severely the economical outcome.

\section{Acknowledgements}

The skilful technical assistance of Dr. R. Rodriguez is very much appreciated. This work was supported by Comisión Interministerial de Ciencia y Tecnología (grant no. IFD 1997-1780 cofinanced with FEDER funds from EU), IFAPA (CO3-125) and Plan Andaluz de Investigación (group no. CVI131), Spain. 


\section{References}

1. Becker, 1994 E.W. Becker

Microalgae: Biotechnology and Microbiology

Cambridge University Press, Cambridge (1994) pp. 293

2. Ben-Amotz, 1995 A. Ben-Amotz

New mode of Dunaliella biotechnology: two-phase growth for $\beta$-carotene production

J. Appl. Phycol., 7 (1995), pp. 65-68

3. Ben-Amotz, 1999 A. Ben-Amotz

Dunaliella $\beta$-carotene: from science to commerce

J. Seckbach (Ed.), Enigmatic Microorganisms and Life in Extreme Environments, Kluwer Academic Publisher, The Netherlands (1999), pp. 401-410

4. Ben-Amotz, 2009 A. Ben-Amotz

Bioactive compounds: glycerol production, carotenoid production, fatty acids production

A. Ben-Amotz, J.E.W. Polle, D.V. Subba Rao (Eds.), The Alga Dunaliella, Biodiversity, Physiology, Genomics and Biotechnology, Science Publishers, Enfield, USA (2009), pp. 189-208

5. Ben-Amotz and Avron, 1983 A. Ben-Amotz, M. Avron

On the factors which determine the massive $\beta$-carotene accumulation in the halotolerant alga Dunaliella bardawil

Plant Physiol., 72 (1983), pp. 593-597

6. Ben-Amotz and Shaish, 1992 A. Ben-Amotz, A. Shaish

$\beta$-Carotene bio-synthesis

M. Avron, A. Ben-Amotz (Eds.), Dunaliella: Physiology, Biochemistry and Biotechnology, CRC Press, Boca Raton, FL (1992), pp. 206-216

7. Ben-Amotz et al., 1988 A. Ben-Amotz, A. Lers, M. Avron

Stereoisomers of $\beta$-carotene and phytoene in the alga Dunaliella bardawil

Plant Physiol., I86 (1988), pp. 1286-1291

8. Borowitzka, 1992 L. Borowitzka

Commercial Dunaliella production: history of development 
T.G. Villa, J. Abalde (Eds.), Profiles on Biotechnology, Universidade de Santiago, A Coruña, Spain (1992), pp. 233-245

9. Borowitzka, 1995 M.A. Borowitzka

Microalgae as sources of pharmaceuticals and other biologically active compounds

J. Appl. Phycol., 7 (1995), pp. 3-15

10. Borowitzka, 1999 M.A. Borowitzka

Commercial production of microalgae:ponds, tubes and fermenters

J. Biotechnol., 70 (1999), pp. 313-321

11. Borowitzka and Borowitzka, 1988 M.A. Borowitzka, L.J. Borowitzka

Dunaliella

M.A. Borowitzka, L.J. Borowitzka (Eds.), Micro-algal Biotechnology, Cambridge University Press, Cambridge, UK (1988), pp. 27-58

12. Del Campo et al., 2001 J.A. Del Campo, H. Rodriguez, J. Moreno, M.A. Vargas, J. Rivas, M.G. Guerrero

Lutein production by Muriellopsis sp. in an outdoor tubular photobioreactor

J. Biotechnol., 85 (2001), pp. 289-295

13. Del Campo et al., 2007 J.A. Del Campo, M. García-González, M.G. Guerrero

Outdoor cultivation of microalgae for carotenoid production: current state and perspectives

Appl. Microbiol. Biotechnol., 74 (2007), pp. 1163-1174

14. Edge et al., 1997 R. Edge, D.J. McGarvey, T.G. Truscott

The carotenoids as antioxidants, a review

J. Photochem. Photobiol., 41 (1997), pp. 189-200

15. García-González et al., 2005 M. García-González, J. Moreno, J.C. Manzano, F.J. Florencio, M.G. Guerrero

Production of Dunaliella salina biomass rich in 9-cis- $\beta$-carotene and lutein in a closed tubular photobioreactor

J. Biotechnol., 115 (2005), pp. 81-90

16. Gómez and González, 2005 P. Gómez, M. González

The effect of temperature and irradiance on the growth and carotenogenic capacity of seven strains of Dunaliella salina (Chlorophyta) cultivated under laboratory conditions 
Biol. Res., 38 (2005), pp. 151-162

17. Guillard and Ryther, 1962 R.R.L. Guillard, J.H. Ryther

Studies of marine plankton diatoms

J. Microbiol., 8 (1962), pp. 229-239

18. Hejazi et al., 2003 M.A. Hejazi, E. Anddrysiewicz, J. Tramper, R.H. Wijffels

Effect of mixing rate on $\beta$-carotene production and extraction by Dunaliella salina in two-phase bioreactor

Biotecnhol. Bioeng., 84 (2003), pp. 591-596

19. Jimenez and Pick, 1994 C. Jiménez, U. Pick

Differential stereoisomer compositions of $\beta$-carotene in thylakoids and in pigments globules in Dunaliella

J. Plant Physiol., 143 (1994), pp. 257-263

20. Johnson and Schroeder, 1995 E.A. Johnson, W.A. Schroeder

A. Fiechter (Ed.), Advances in Biochemical Engineering Biotechnology, vol. 53Springer-Verlag, Berlin (1995), pp. 119-178

21. Lee, 2001 Y.K. Lee

Microalgal mass culture systems and methods: their limitation and potential

J. Appl. Phycol., 13 (2001), pp. 307-315

22. Liu et al., 2000 Q. Liu, K. Suzuki, S. Nakaji, K. Sugawara

Antioxidant activities of natural 9-cis and synthetic all-trans $\beta$-carotene assessed by human neutrophil chemiluminescence

Nutr. Res., 20 (2000), pp. 5-14

23. Orset and Young, 2000 S. Orset, A. Young

Exposure to low irradiances favors the synthesis of 9-cis- $\beta$-carotene in Dunaliella salina (Teod.)

Plant Physiol., 122 (2000), pp. 609-617

24. Palozza and Krinsky, 1992 P. Palozza, N.I. Krinsky

Antioxidant effects of carotenoids in vivo and in vitro: an overview

Methods Enzymol., 213 (1992), pp. 403-420

25. Phillips et al., 1995 L.G. Phillips, A.K. Cowan, P.D. Rose, M.R.R. Logie 
Operation of the xanthophylls cycle in non-stress and stressed cells of Dunaliella salina in response to diurnal changes in incident irradiation: a correlation with intracellular $\beta$-carotene content

J. Plant Physiol., 146 (1995), pp. 547-553

26. Richmond, 1991 A. Richmond

Large-scale microalgal culture and applications

Round, Chapman (Eds.), Progress in Phycological Research, Biopress Ltd., Bristol, England (1991) pp. 1-62

27. Richmond et al., 1993 A. Richmond, S. Boussiba, A. Vonshak, R. Kopel

A new tubular reactor for mass production of microalgae outdoors

J. Appl. Phycol., 5 (1993), pp. 327-332

28. Shaish et al., 1992 A. Shaish, A. Ben-Amotz, M. Avron

Biosynthesis of $\beta$-carotene in Dunaliella

Methods Enzymol., 213 (1992), pp. 439-444

29. Tredici, 2004 M.R. Tredici

Mass production of microalgae: photobioreactors

A. Richmond (Ed.), Handbook of Microalgal Culture: Biotechnology and Applied PhycologyBlackwell, Oxford, UK (2004), pp. 178-214

30. Tredici and Zitelli, 1997 M.R. Tredici, G.C. Zitelli

Cultivation of Spirulina (Arthrospira) platensis in flat plate reactor

A. Vonshak (Ed.), Spirulina platensis (Arthrospira)- Physiology, Cell Biology and Biotechnology, Tailor and Francis, London, U.K. (1997) pp. 117-130

31. Wijffels et al., 2009 R.H. Wijffels, M. Barbosa, M. Jansen

Cultivation of Dunaliella for high value compounds

A. Ben-Amotz, J.E.W. Polle, D.V. Subba Rao (Eds.), The Alga Dunaliella, Biodiversity, Physiology, Genomics and Biotechnology, Science Publishers, Enfield, USA (2009), pp. 91-110 


\section{Figure captions}

Figure 1. Outdoor cultivation of Dunaliella salina under batch (A) and semi continuous (B) regime in $3 \mathrm{~m} 2$ open tanks. The culture corresponds to a month of spring.

Figure 2. Aggregated monthly carotenoid production by Dunaliella salina cultures in $3 \mathrm{~m} 2$ open tanks performed under batch or semi continuous regime and closed tubular photobioreactor under batch regime. Mean \pm SD of experimental data for several years were assembled by seasons.

Figure 3. Outdoor cultivation of Dunaliella salina under batch (A) and semi continuous (B) regime in closed tubular photobioreactor. The culture corresponds to a month of spring. 
Table 1. Carotenoid production by outdoor cultures of $D$. salina under batch, semi-continuous and two-stage regime in open tank and closed tubular photobioreactor.

\begin{tabular}{|c|c|c|c|c|c|c|}
\hline & \multicolumn{3}{|c|}{ Open tank } & \multicolumn{3}{|c|}{ Closed tubular reactor } \\
\hline & Batch & $\begin{array}{l}\text { Semi- } \\
\text { continuous }\end{array}$ & $\begin{array}{l}\text { Two } \\
\text { stage }\end{array}$ & Batch & $\begin{array}{l}\text { Semi- } \\
\text { continuous }\end{array}$ & $\begin{array}{l}\text { Two } \\
\text { stage }\end{array}$ \\
\hline $\begin{array}{|lr|}\begin{array}{l}\text { Maximun } \\
\text { concentration } \\
\text { culture })\end{array} & \quad \mathrm{mg} \mathrm{l}^{-1} \\
\end{array}$ & 8.9 & 3.8 & 14.3 & 62.2 & 50.0 & 82.2 \\
\hline Cycles per month & 2 & 6 & 3 & 3 & 5 & 4 \\
\hline $\begin{array}{|lr|}\text { Aggregated } & \text { monthly } \\
\text { carotenoid } & \text { production } \\
\left(\mathrm{mg} \mathrm{l}^{-1} \text { culture }\right)\end{array}$ & 17.8 & 22.9 & 42.9 & 186.5 & $\mid 250.0$ & 328.8 \\
\hline
\end{tabular}

Data shown are average of experiments carried out several years and correspond to maximal values obtained in spring (irradiance, $10 \mathrm{MJ} \mathrm{m}^{-2} \mathrm{~d}^{-1}$ ). Nitrate concentration in the batch cultures was $5 \mathrm{mM}$ at the beginning of the culture; in semi-continuous culture the nitrate concentration was maintained between 0.5 and $3 \mathrm{mM}$. In the second phase of the two-stage approach, nitrate is undetectable. 
Table 2. Effect of the outdoor impinging radiation on the carotenoid content of D. salina cultures in open tanks during the second stage of two-stage batch cultures.

\begin{tabular}{|c|c|c|}
\hline $\begin{array}{l}\text { Irradiance } \\
\left(\mathrm{MJ} \mathrm{m}^{-2} \mathrm{~d}^{-1}\right)\end{array}$ & \begin{tabular}{|l} 
Carotenoids \\
$\left(\mathrm{mg} \mathrm{l}^{-1}\right.$ culture $)$
\end{tabular} & \begin{tabular}{|llll}
$\begin{array}{l}\text { Carotenoids } \\
\text { biomass }\end{array}$ & $(\%$ of & dry \\
\end{tabular} \\
\hline 3 & $4.7 \pm 0.24$ & 6.0 \\
\hline 6 & $8.6 \pm 0.12$ & 7.4 \\
\hline 10 & $14.3 \pm 0.53$ & 9.7 \\
\hline
\end{tabular}

Biomass transferred to production tanks (time 0) held about $1.5 \mathrm{mg}$ carotenoids $\mathrm{l}^{-1}$ of culture (4\% of dry biomass). Determinations were performed in samples taken at the 6th day of maintenance of the cultures in the production tanks on a medium lacking added nitrogen. Data are mean \pm SD based on monthly values over a two years period. 
Table 3

Table 3. Carotenoid composition of $D$. salina cultures in open tanks and tubular reactor in the second phase of two-stage culture carried out in spring $\left(10 \mathrm{MJ} \mathrm{m}^{-2} \mathrm{~d}^{-1}\right)$.

\begin{tabular}{|l|l|l|}
\hline & Open tanks & Closed tubular reactor \\
\hline \hline -Carotene & $80 \%$ & $90 \%$ \\
\hline Lutein & $4 \%$ & $5 \%$ \\
\hline 9-Cis/all trans $\beta$-carotene ratio & $1.01 \pm 0.05$ & $1.53 \pm 0.08$ \\
\hline
\end{tabular}

a Data at sunrise at the end of the second phase (nitrate is undetectable). 
Figure 1

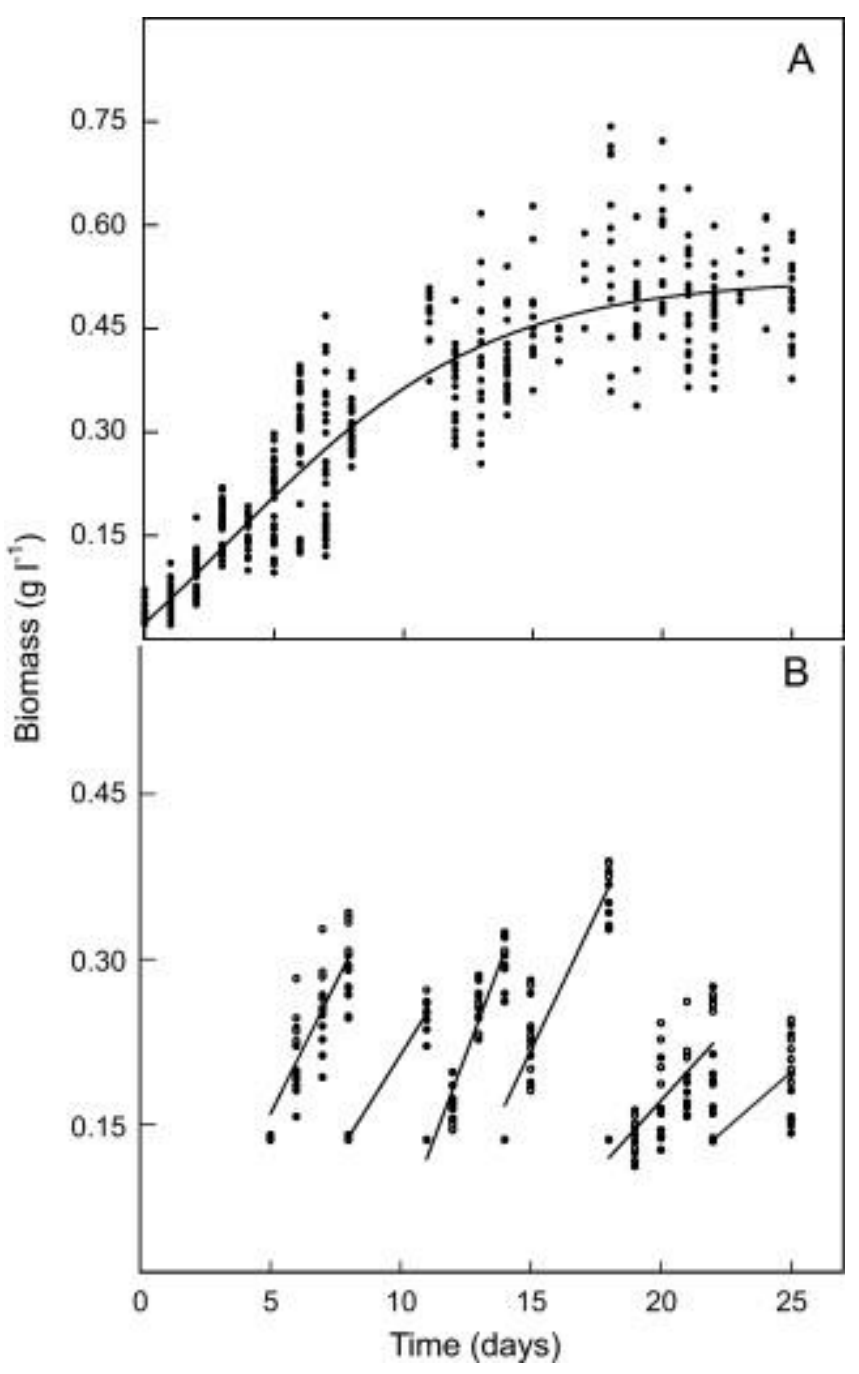


Figure 2

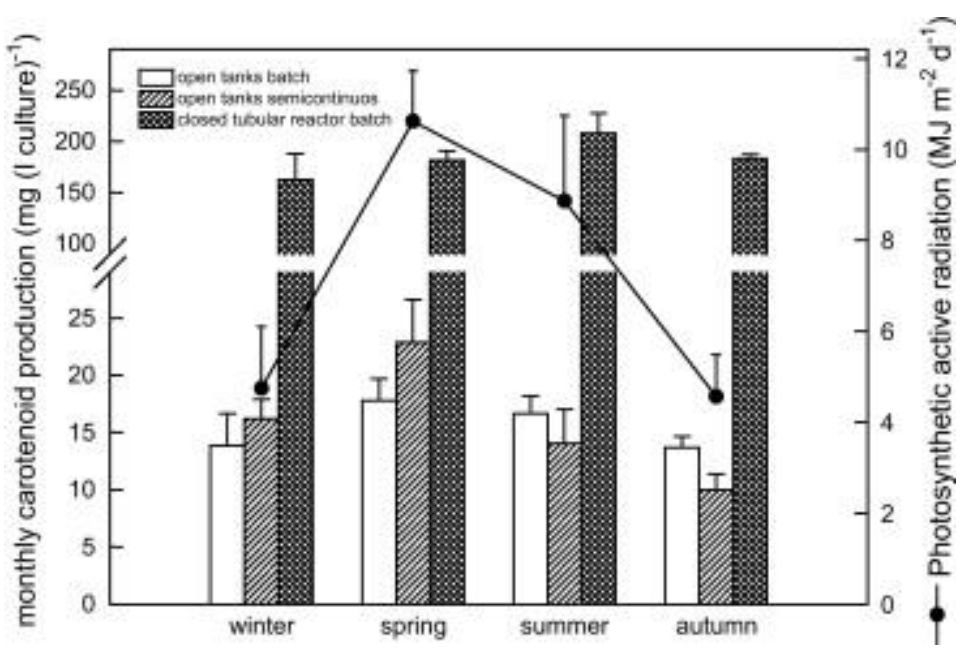


Figure 3

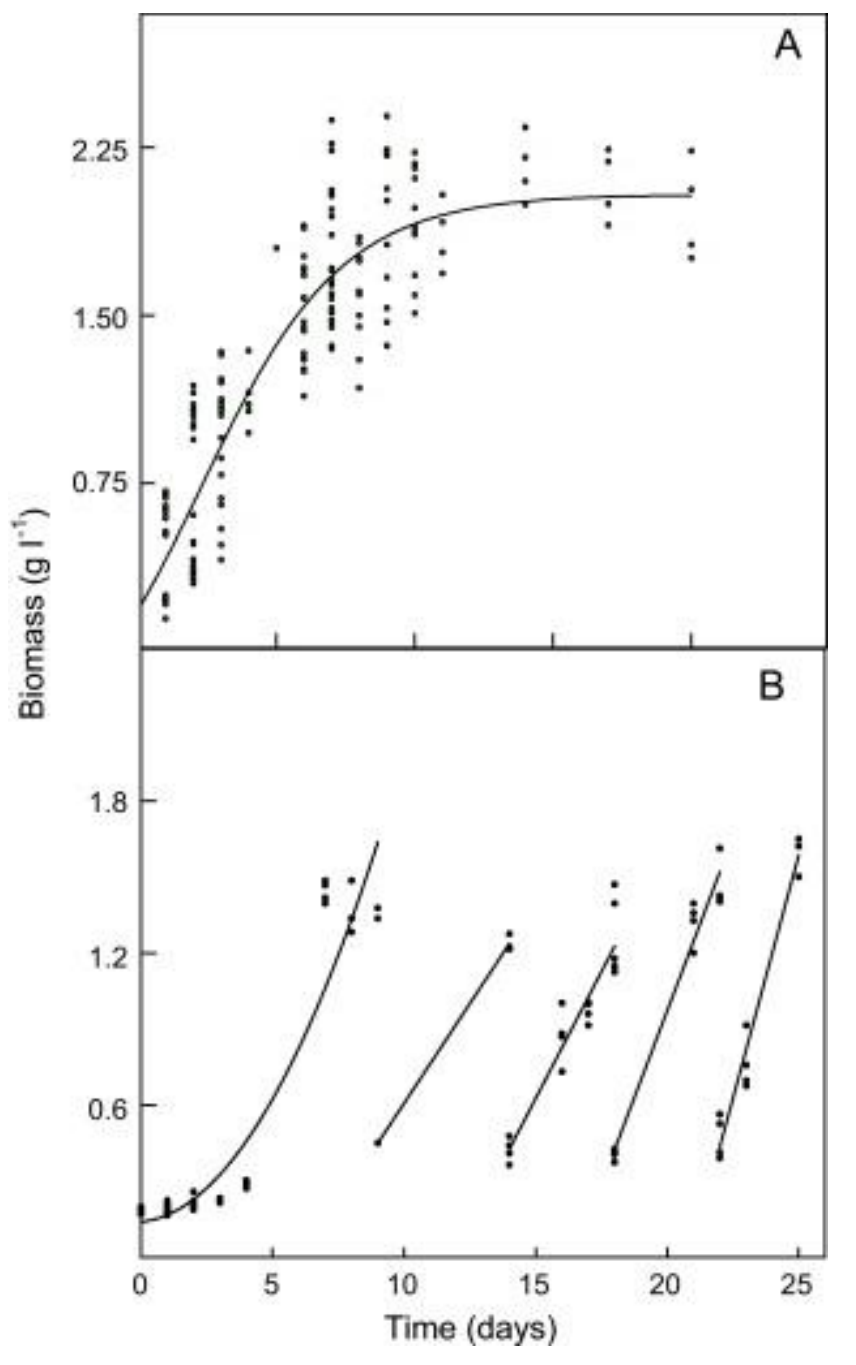

